\title{
Brain Mechanisms Supporting Spatial Discrimination of Pain
}

\author{
Yoshitetsu Oshiro, ${ }^{1}$ Alexandre S. Quevedo, ${ }^{1}$ John G. McHaffie, ${ }^{1}$ Robert A. Kraft, ${ }^{2}$ and Robert C. Coghill ${ }^{1,3}$ \\ ${ }^{1}$ Department of Neurobiology and Anatomy, ${ }^{2}$ Department of Biomedical Engineering, and ${ }^{3}$ Center for the Study of Pharmacologic Plasticity in the Presence \\ of Pain, Wake Forest University School of Medicine, Winston-Salem, North Carolina 27157-1010
}

\begin{abstract}
Pain is a uniquely individual experience that is heavily shaped by evaluation and judgments about afferent sensory information. In visual, auditory, and tactile sensory modalities, evaluation of afferent information engages brain regions outside of the primary sensory cortices. In contrast, evaluation of sensory features of noxious information has long been thought to be accomplished by the primary somatosensory cortex and other structures associated with the lateral pain system. Using functional magnetic resonance imaging and a delayed match-to-sample task, we show that the prefrontal cortex, anterior cingulate cortex, posterior parietal cortex, thalamus, and caudate are engaged during evaluation of the spatial locations of noxious stimuli. Thus, brain mechanisms supporting discrimination of sensory features of pain extend far beyond the somatosensory cortices and involve frontal regions traditionally associated with affective processing and the medial pain system. These frontoparietal interactions are similar to those involved in the processing of innocuous information and may be critically involved in placing afferent sensory information into a personal historical context.
\end{abstract}

Key words: dorsolateral prefrontal cortex; anterior cingulate cortex; somatosensory; perceptual judgments; memory; evaluation

\section{Introduction}

Pain is a private, personal experience that is unique from one individual to the next (Coghill et al., 2003). Cognitive evaluation of afferent nociceptive information contributes substantially to the elaboration of this sensory information into a uniquely individual experience of pain by placing this information into a personal context. In other sensory modalities, subjective awareness, evaluation, and decisions about sensory information involve brain regions that extend far beyond the primary sensory cortices (Crick and Koch, 1995; Bushara et al., 1999; Romo and Salinas, 2003; Heekeren et al., 2004). For example, until neurons in the frontal cortex become active, monkeys do not report a subjective experience of a vibratory stimulus that is sufficient to activate neurons in the primary somatosensory cortex (SI) (de Lafuente and Romo, 2005).

Despite this evidence from other sensory modalities, current conceptual models of pain processing restrict discriminative processing of sensory information to the "lateral pain system" (Melzack and Casey, 1968; Treede et al., 1999). The lateral pain system consists of SI and the secondary somatosensory cortex (SII) and the ventroposterior lateral and medial nuclei of the thalamus. In contrast, brain regions belonging to the "medial pain system," such as the prefrontal cortex and anterior cingulate cortex (ACC), are thought to be involved primarily with affective-

Received Nov. 27, 2006; revised Feb. 12, 2007; accepted Feb. 15, 2007.

This work was supported by National Institutes of Health Grant R01 NS39426. We thank the FMRIB Image Analysis Group (Oxford University, Oxford, UK) for the FSL analysis software and Emilio Salinas for his helpful comments on this manuscript.

Correspondence should be addressed to Dr. Robert C. Coghill, Department of Neurobiology and Anatomy, Wake Forest University School of Medicine, Winston-Salem, NC 27157-1010. E-mail: rcoghill@wfubmc.edu. DOI:10.1523/JNEUROSCI.5128-06.2007

Copyright $\odot 2007$ Society for Neuroscience $\quad 0270-6474 / 07 / 273388-07 \$ 15.00 / 0$ motivational aspects of pain (Melzack and Casey, 1968; Treede et al., 1999). However, several indirect lines of evidence suggest that brain regions outside the lateral pain system may contribute importantly to the evaluation of pain. First, anecdotal observations of patients with prefrontal lobotomies suggest that the prefrontal cortex is important both in the evaluation of pain and in applying a contextual significance to afferent nociceptive information (Freeman and Watts, 1948). Second, regions of the prefrontal cortex have been reported to exhibit nonlinear stimulus-response curves while subjects are rating graded intensities of noxious stimuli. Because activation peaks near pain threshold in which subjective ratings become most demanding, these regions are suggested to be involved with the evaluation of pain (Coghill et al., 1999). Finally, noxious stimuli produce robust activation of the SI but not the prefrontal cortex or ACC in patients in persistent vegetative states (Laureys et al., 2002). Because these individuals are thought to have no conscious experience of pain or other stimuli, coactivation of structures outside of the lateral pain system appears critical for generating a subjectively available experience of pain (Laureys et al., 2002).

Despite the profound importance of understanding processes involved in the evaluation of pain, no direct evidence has yet determined which brain mechanisms support discrimination of sensory features of pain. To identify brain regions specifically associated with the evaluation of sensory features of nociceptive information, we recruited subjects to undergo functional brain imaging while they evaluated the spatial location of noxious stimuli.

\section{Materials and Methods}

Subjects. This study was performed on 12 healthy volunteers, six females and six males (age, 22-39 years; mean, 29 years). Six subjects were White 


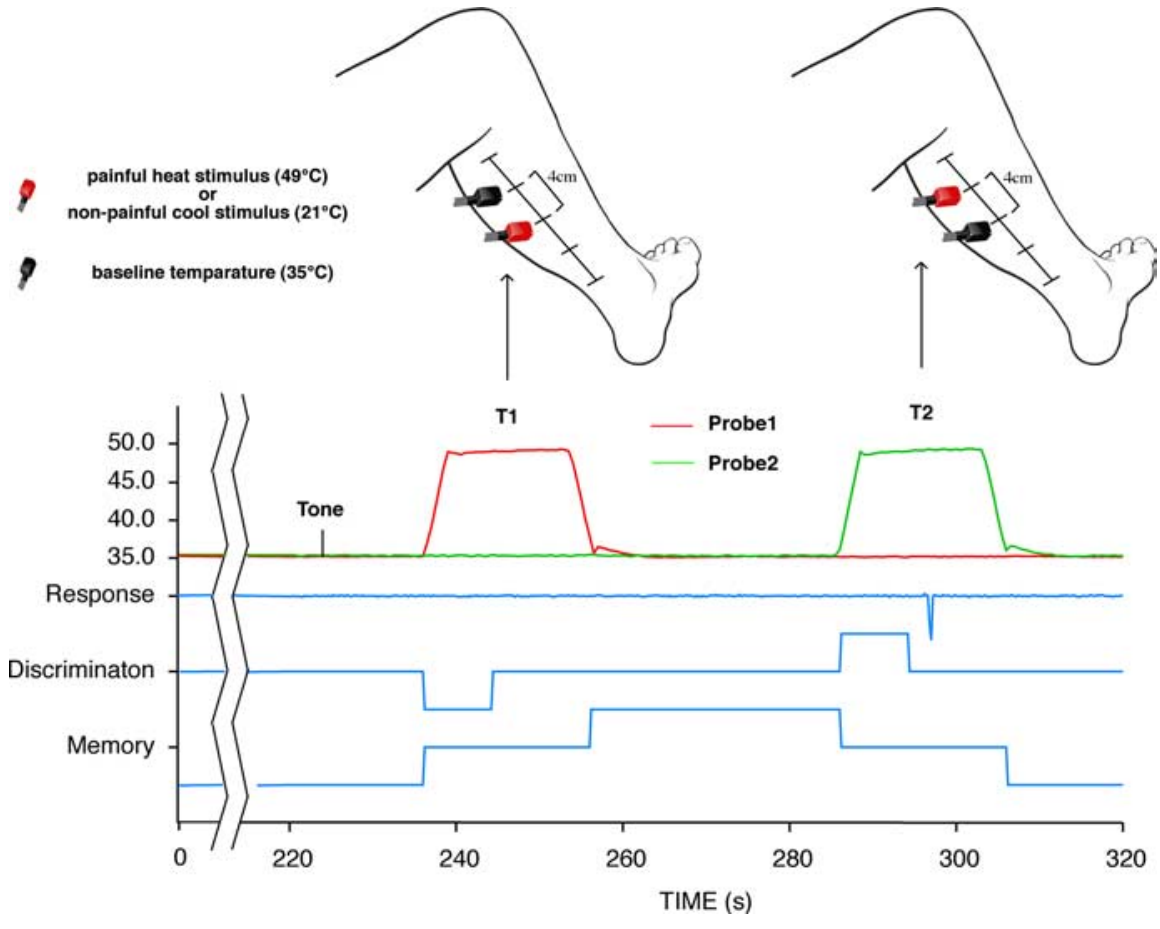

Figure 1. The temporal sequence of the discrimination task. Noxious heat (or innocuous cool) stimuli were delivered sequentially to the back of the lower leg via two thermal probes separated by varying distances. Before the end of $T 2$, subjects had to indicate whether T2 was in a different (or same) spatial location as T1. Discrimination-related brain activity was identified using regressors determined by response latencies in each individual discrimination trial. Additional regressors were used to identify brain activation that was related to spatial memory and to perceived pain (or cool).

(three males, three females), four subjects were Asian (three males, one female), and two were Black (two females). All subjects gave written, informed consent acknowledging that (1) they would experience experimental painful stimuli, (2) all methods and procedures were clearly explained, and (3) they were free to withdraw from the experiment at any time without prejudice. All of the procedures were approved by the Institutional Review Board of Wake Forest University School of Medicine.

Psychophysical training. All subjects first received $325 \mathrm{~s}$ duration stimuli $\left(35-49^{\circ} \mathrm{C}\right)$ to give them experience rating pain. Next, they received the exact stimulus paradigms that were used during the functional magnetic resonance imaging (fMRI) session to ensure that they could adequately perform the discrimination task. Additionally, this procedure served to familiarize them with the temporal sequence of stimuli within a series to minimize variations in cognitive components such as expectation and anxiety.

Experimental task. A two-alternative forced-choice paradigm using pairs of thermal stimuli was used to identify brain regions involved in the processing of spatial discrimination of pain (Fig. 1). Two thermal stimulators (TSA II, $16 \times 16 \mathrm{~cm}$ surface area each, rise/fall rates of $6^{\circ} \mathrm{C} / \mathrm{s}$; Medoc, Ramat Yishai, Israel) were placed on the lower left leg of each subject and separated by distances of 4,8 , and $16 \mathrm{~cm}$. After a $30 \mathrm{~s}$ rest period $\left(35^{\circ} \mathrm{C}\right)$, one of the two was activated for $20 \mathrm{~s}\left[49^{\circ} \mathrm{C}\right.$; stimulus 1 (T1)], and after a $30 \mathrm{~s}$ working memory interval (both stimulators at $\left.35^{\circ} \mathrm{C}\right)$, one of the two stimulators was again activated $\left[49^{\circ} \mathrm{C}\right.$ for $20 \mathrm{~s}$; stimulus 2 (T2)]. Subjects were required to signal if the location of T2 was the same as (key press with the right index finger) or different from (key press with the right middle finger) T1. An important aspect of this paradigm is that subjects were required to signal their decision as soon as the determination was made and before the termination of T2. Responses were recorded on a digital chart recorder (PowerLab; ADInstruments, Colorado Springs, CO) and subsequently incorporated into the fMRI analysis. Three discrimination pairs (i.e., six stimuli) were delivered in each fMRI series, with the beginning of each discrimination pair signaled with a $1 \mathrm{~s}$ tone $5 \mathrm{~s}$ before the onset of T1. At the end of each series, subjects rated pain intensity, pain unpleasantness, and subjective task difficulty on visual analog scales (Price et al., 1994). Subjects were also queried for the strategy that they used to remember and evaluate the spatial location of the stimuli. An identical spatial discrimination task using pairs of innocuous cool stimuli $\left(21^{\circ} \mathrm{C} ; 4 \mathrm{~cm}\right.$ separation) was used to determine whether similar brain networks were involved in discriminating the location of nonpainful stimuli. All series of stimuli were delivered in a randomized order.

Statistical analysis of psychophysical data. To examine the effects of separation distance on the difficulty of spatial discrimination, we calculated response latencies and error rates. These, together with subjective ratings of task difficulty and pain intensity, were examined with an ANOVA repeated within subjects over probe separation distance (JMP software; SAS Institute, Cary, NC).

Image acquisition and image processing. $\mathrm{fMRI}$ data were acquired on a $1.5 \mathrm{~T}$ General Electric Twin-Speed LX Scanner with a birdcage quadrature head coil (General Electric Medical Systems, Milwaukee, WI). For functional imaging, two-dimensional blood oxygenation leveldependent images of the entire brain were acquired continuously by using single-shot echoplanar imaging [echo time (TE), $40 \mathrm{~ms}$; repetition time (TR), $2 \mathrm{~s} ; 28 \times 5$-mm-thick slices; in-plane resolution, $3.72 \times 3.75 \mathrm{~mm}$; flip angle, $90^{\circ}$; no slice gap] (Ogawa et al., 1990). Each fMRI series was $300 \mathrm{~s}$ long and consisted of 150 volumes. A total of $12 \mathrm{fMRI}$ series were obtained for each subject, with 9 series examining pain discrimination and 3 series examining discrimination of nonpainful stimuli. Different stimulus separation distances and stimulus temperatures were presented in random order across series. During fMRI acquisition series, subjects were requested to close their eyes. Tones that cued initiation of each discrimination trial were delivered through MRI-compatible headphones. High-resolution structural scans were acquired using a three-dimensional (3D) spoiled gradient-echo (3D inversion spoiled gradient-recalled acquisition in a steady state) sequence (inversion time, $600 \mathrm{~ms}$; TR, $9.1 \mathrm{~ms}$; flip angle, $20^{\circ}$; TE, $1.98 \mathrm{~ms}$; matrix, $256 \times 196$; section thickness, $1.5 \mathrm{~mm}$ with no gap between sections; 124 sections; in-plane resolution, $0.9375 \times 0.9375$ $\mathrm{mm}$; field of view, $24 \mathrm{~cm}$ ).

The functional image analysis package FSL [Functional Magnetic Resonance Imaging of the Brain (FMRIB) Software Library (Center for FMRIB, University of Oxford, Oxford, UK)] was used for image processing and statistical analysis. The functional data were movement corrected, spatially smoothed by $5 \mathrm{~mm}$ with a 3D isotropic Gaussian kernel, and temporally filtered by a nonlinear high-pass filter with a cutoff period of 75 s. Each functional image was scaled by its mean global intensity (intensity normalization). Next, each subject's functional images were registered to their structural data using a seven-parameter linear 3D transformation and transformed into standard stereotaxic space (as defined by the Montreal Neurological Institute) using a 12-parameter linear 3D transformation (Talairach and Tournoux, 1988; Jenkinson et al., 2002).

Statistical analysis of regional signal changes within the brain. Separate predictive model functions were derived to assess brain activation during discrimination, memory, and thermal stimulation (Fig. 1). To examine discrimination-related activation, response latencies for each discrimination were calculated. The time period from $\mathrm{T} 2$ onset to $75 \%$ of the response latency was weighted as +1 , whereas the corresponding time period during $\mathrm{T} 1$ was weighted as -1 . Thus, this regressor compares activity during a time period of active discrimination of painful (or cool) stimulation with a period of equal intensity stimulation while no discrimination was being actively performed. Accordingly, this regressor identifies the brain activity mediating the evaluation of location differ- 
ences. However, it does not allow the identification of brain mechanisms that contribute to basic awareness of the spatial location of a single stimulus, because these mechanisms would be equally active during both $\mathrm{T} 1$ and T2. To ensure that motor activity resulting from response selection did not confound assessment of discrimination activation, only the first $75 \%$ of the discrimination period was examined. Factors independent of discrimination could also lead to greater (or lesser) activity in T2 versus T1. For example, a progressive sensitization over all six stimuli in a series would cause all time points of T2 to be greater than T1. In such a case, activation in the postdecision period would be elevated in a manner like that of the predecision period. Thus, to further assess the specificity of discrimination-related activation, activity during the postdecision period of T2 was compared with that of the analogous time period of T1 in a manner identical to that of the discrimination period. To examine memory-related activation, a simple boxcar function was used to compare activation during the memory interval with that of nonstimulated rest periods. All stimuli in a given MRI series were the same intensity, and the interstimulus intervals (ISIs) between $\mathrm{T} 1$ and $\mathrm{T} 2$ were equal to the ISIs between T2 and the next T1. Thus, variations in other cognitive functions were minimized, and the effort to memorize the location is likely to be the dominant process contributing to activation in the period between T1 and T2 (vs the period between T2 and the next T1). Pain- and cool-related activation were also examined using simple boxcar functions. All of the regressors were convolved with a gamma-variate model of the hemodynamic response (delay, $6 \mathrm{~s}$; SD, $3 \mathrm{~s}$ ) and its temporal derivative and were temporally filtered with the same parameters as the fMRI data. Because discrimination regressors varied with response latency, this analysis is effectively weighted by discrimination difficulty. Thus, a separate analysis for each separation distance was not performed. Using these regressors, interseries group analyses across 12 subjects were performed separately for memory, discrimination, and pain (or cool), with fixed-effects models within series and within-subject and randomeffects models between subjects (Woolrich et al., 2001). Clusters of voxels exceeding a $Z$ score $>2.3$ and $p<0.05$ were considered statistically significant (Worsley et al., 1992).

\section{Results}

\section{Psychophysical and behavioral results}

During the pain discrimination paradigm, all indices of discrimination difficulty increased with increasing proximity of the two thermal stimuli (response latency: overall $F=65.51, p<0.0001$; 4 vs $8 \mathrm{~cm}, F=6.30, p<0.029 ; 4$ vs $16 \mathrm{~cm}, F=37.26, p<0.0001$; error rate: overall $F=9.99, p<0.0001 ; 4$ vs $8 \mathrm{~cm}, F=23.57, p<$ 0.0005 ; 4 vs $16 \mathrm{~cm}, F=10.09, p<0.0088$; subjective difficulty: overall $F=19.13, p<0.0001 ; 4$ vs $8 \mathrm{~cm}, F=21.47, p<0.0007 ; 4$ vs $16 \mathrm{~cm}, F=35.60, p<0.0001$ ) (Fig. 2). These findings confirm that subjects were correctly performing the discrimination task. Pain-intensity ratings remained primarily stable across all stimulus separation distances $(F=1.65 ; p<0.2147)$, although a trend toward decreasing ratings was observed with increasing task difficulty (4 vs $16 \mathrm{~cm}: F=3.53, p<0.0868$ ) (Fig. 2).

Response latencies during cool discrimination were significantly shorter than those during heat pain discrimination $(F=$ $182.48 ; p<0.0001)$. However, the error rates $(F=3.87$; $p<$ $0.075)$ and perceived difficulty $(F=1.16 ; p<0.3035)$ of cool discrimination were not reliably different from those of pain discrimination. Thus, difficulty was kept approximately constant across pain and cool discrimination tasks, and, as such, the cool task serves as an appropriate control condition. Different perceptual time courses may account for the different response latencies between pain and cool discrimination decisions. Psychophysical pilot data indicate that subjects adapt to innocuous cool stimulation relatively rapidly. In contrast, pain from $49^{\circ} \mathrm{C}$ tends to progressively summate over the course of the $20 \mathrm{~s}$ stimulation period. Thus, subjects have the most information on which to
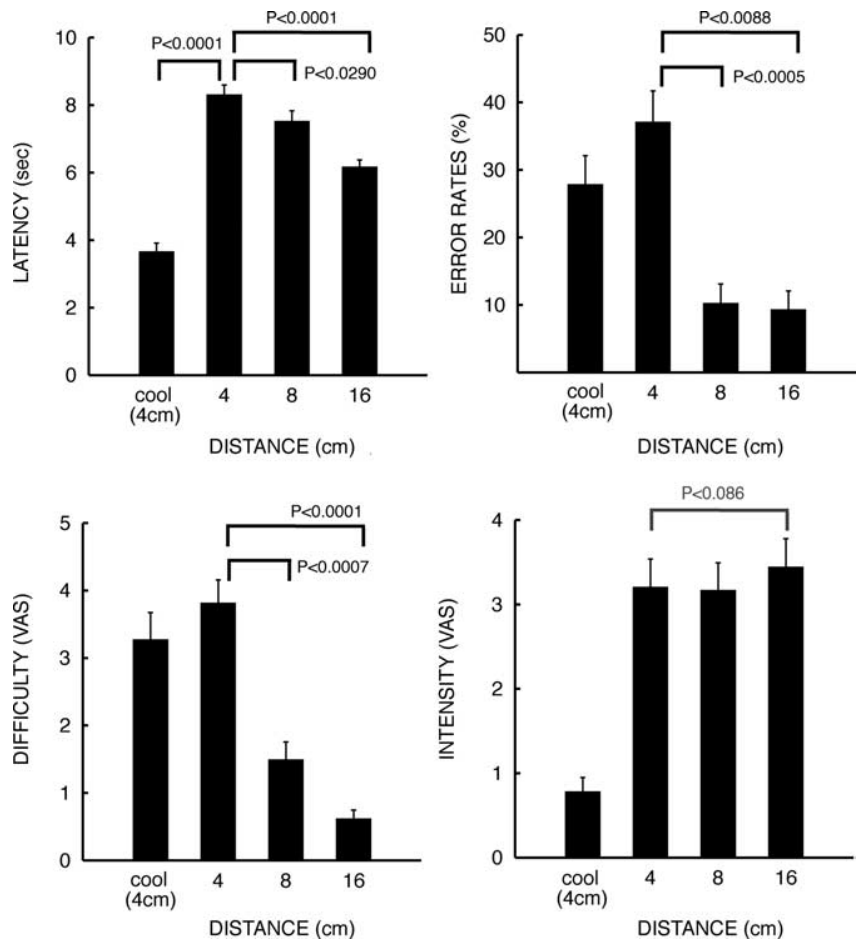

Figure 2. Behavioral responses during spatial discrimination (means \pm SEM). Discrimination of noxious heat became progressively more difficult with increasing probe proximity $(16,8$, $4 \mathrm{~cm}$ ). Response latencies, error rates, and subjective ratings of perceived difficulty increased monotonically as stimuli were delivered closer together. In contrast, perceived pain intensity exhibited a trend toward decreasing with increasing stimulus proximity. At $4 \mathrm{~cm}$ separation distances, cool discrimination was performed more rapidly than noxious heat discrimination but was nearly equally difficult. VAS, Visual analog scale.

make a decision during the early period of cool stimulation and during the late period of heat pain stimulation.

\section{Pain and innocuous cool-related activation}

During painful heat stimulation of the lower left leg, paininduced brain activation was identified within bilateral portions of the cerebellum, thalamus, caudate, putamen/globus pallidus, frontal operculum, anterior insula, posterior insula/SII, ACC, and supplementary motor area (Fig. 3) (supplemental Table 1, available at www.jneurosci.org as supplemental material). These results are consistent with previous studies and indicate that the subjects were sufficiently sensitive and that the stimuli were sufficiently robust to reliably detect activation of structures associated with the "medial" and "lateral" pain system (Treede et al., 1999). During innocuous cool stimulation, significant signal changes were evident within brain regions that were similar to those activated during painful heat stimulation (Fig. 4) (supplemental Table 1, available at www.jneurosci.org as supplemental material).

\section{Spatial discrimination-related activation}

During the spatial discrimination task, a widely distributed array of cerebral cortical and subcortical regions was activated while subjects were actively comparing the present location of a painful stimulus with that of a remembered stimulus location. These areas included bilateral portions of the ACC and the posterior parietal cortex (PPC), as well as the right dorsolateral prefrontal cortex (DLPFC), right head of the caudate nucleus, right anterior and right medial thalamus, and left primary motor cortex (Fig. 3, Table 1). In contrast, neither SI nor SII was differentially acti- 


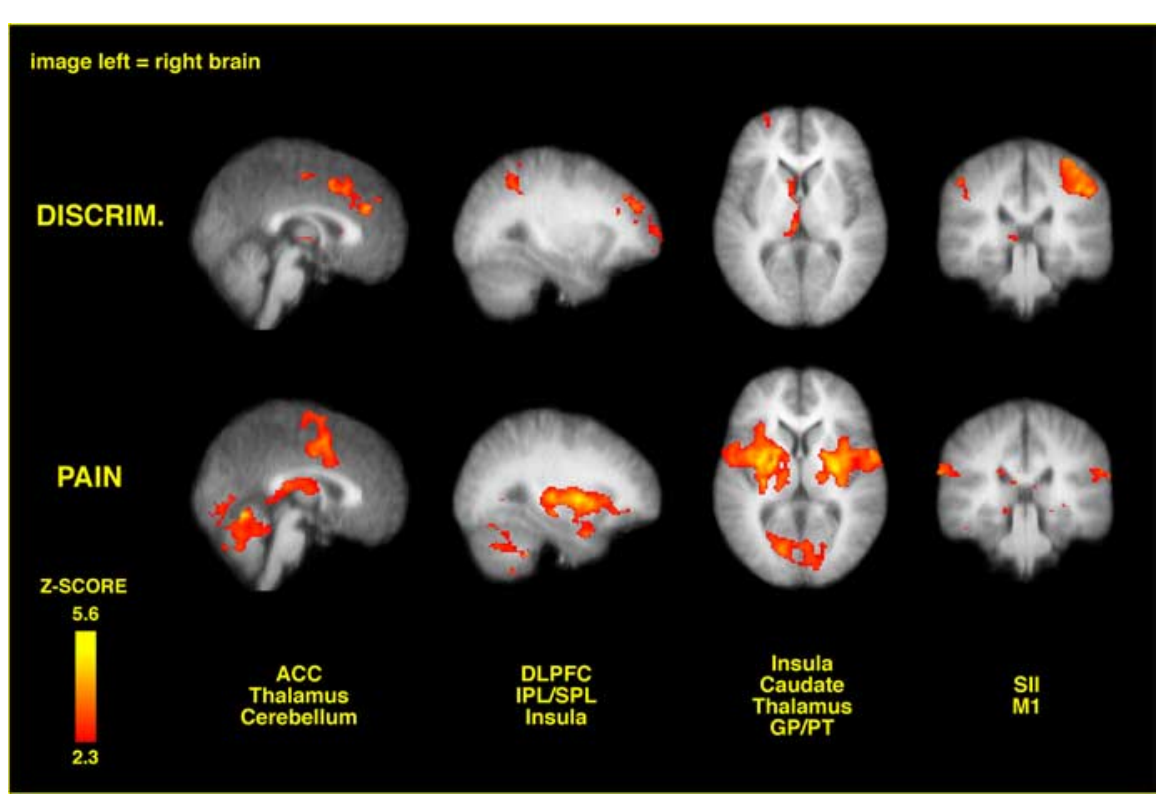

Figure 3. Brain activation related to spatial discrimination of noxious stimuli is distinct from that related to perceived pain. These images are located at $x=0 \mathrm{~mm}, x=30 \mathrm{~mm}, z=5 \mathrm{~mm}$, and $y=-30 \mathrm{~mm}$ in standard stereotaxic space. IPL/SPL, Inferior parietal lobule/superior parietal lobule; GP/PT, globus pallidus/putamen; M1, primary motor cortex; DISCRIM., discrimination.

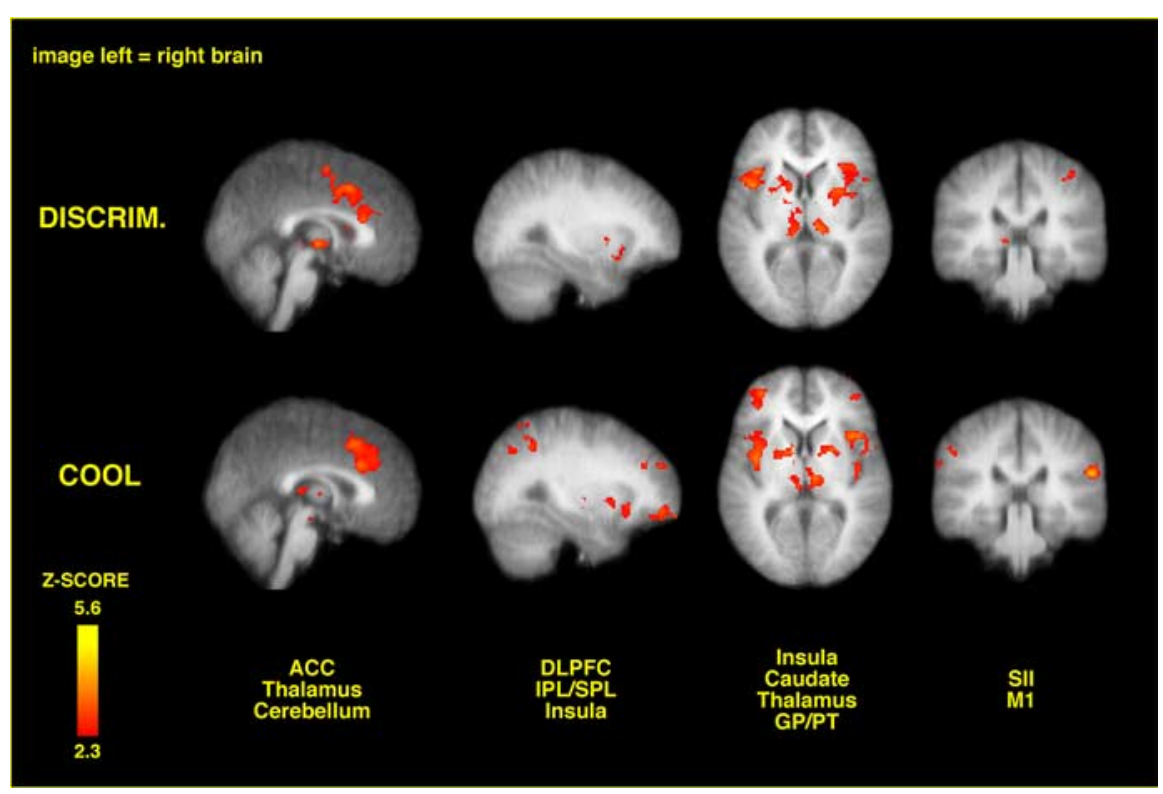

Figure 4. Brain activation during spatial discrimination of innocuous cool stimuli. These images are located at $x=0 \mathrm{~mm}, x=$ $30 \mathrm{~mm}, \mathrm{z}=5 \mathrm{~mm}$, and $y=-30 \mathrm{~mm}$ in standard stereotaxic space. IPL/SPL, Inferior parietal lobule/superior parietal lobule; GP/PT, globus pallidus/putamen; M1, primary motor cortex, DISCRIM., discrimination.

vated during spatial discrimination. However, portions of SII, adjacent frontoparietal operculum, and posterior insula exhibited diminished activity during discrimination (Table 1). During the postdecision period, none of the regions exhibiting activation during the predecision period remained activated.

A generally similar group of brain regions was activated during spatial discrimination of innocuous cool stimuli. These areas include the ACC, thalamus, and caudate nucleus. Additional activation was also noted in the insular cortex. As with spatial discrimination of noxious stimuli, differential activation of SI and SII was not noted (supplemental Table 2, available at www. jneurosci.org as supplemental material) (Fig. 4).

\section{Spatial memory-related activation}

During spatial memory for pain, the most frequent strategy for remembering and evaluating the location of the noxious stimulus involved subjects remembering a verbal descriptor of the stimulus location (Fig. 5). Consistent with this verbal strategy, activation during spatial memory for pain was lateralized to the left hemisphere (Fig. 5, Table 1). Signal changes during the memory period were detected within the left frontal operculum, left inferior frontal cortex, left DLPFC, left inferior parietal lobule, and left premotor cortex. During spatial memory for cool location, similar portions of the left DLPFC were activated. However, robust activation of the ACC was also detected (supplemental Table 2, available at www.jneurosci.org as supplemental material).

During noxious stimulation, spatial discrimination-related activation overlapped minimally with spatial memoryrelated activation. However, during both conditions, statistically reliable activation was detected in the hand regions of the left primary motor cortex as well as related premotor and sensory areas (Fig. 5). Because subjects were required to indicate their discrimination choice by pushing response buttons with fingers on their right hand, this overlapping left motor activation likely represents motor-related processing. Given that no movements were required during the memory period and that discrimination-related brain activity was examined before subjects' responses, activation of these areas likely reflects motor preparation and planning rather than actual movement.

\section{Discussion}

In delayed match-to-sample tasks, the cognitive act of perceptual decision making involves a complex chain of events including stimulus encoding, perceptual memory, and comparisons of previously experienced information with incoming afferent information (Romo and Salinas, 2001). In multiple sensory modalities, brain structures outside the primary sensory cortices have been demonstrated to be critically involved in such decision-making processes (Bushara et al., 1999; Romo and Salinas, 2003; Heekeren et al., 2004). For example, multiple frontal and prefrontal cortical regions are activated during judgments of visual and tactile information (Romo and Salinas, 2003; Heekeren et al., 2004). However, classical views of nociceptive processing hold that discriminative processing of sensory information occurs in the somatosensory cortices, whereas affective processing occurs in areas closely associated with the limbic system (Melzack and Casey, 1968; Treede et al., 1999). The present findings challenge this long-held and widely assumed assertion and indicate that numerous brain 
regions beyond the somatosensory cortices are differentially involved in a fundamental discriminative process-spatial discrimination of pain. Moreover, the present findings confirm that several brain regions traditionally associated with affective processing in the medial pain system are robustly activated during discriminative processing of sensory aspects of pain.

\section{Sensory-discriminative processing in SI} and SII

Despite their crucial connectivity, brain regions associated with the lateral pain system, such as the SI and ventroposteriorlateral thalamus, did not exhibit statistically reliable increases in activity during memory or spatial discrimination. Moreover, portions of SII and posterior insula exhibited less activity during discrimination, a finding potentially related to the trend for decreased reports of pain intensity during more difficult discriminations (Fig. 2). This decreased activation may be related to a progressive reallocation of neural resources from afferent processing to evaluative processes during progressively more difficult discriminations. Alternatively, these decreases may be associated with task-related alterations in the spatial tuning of nociceptive neurons. A reduction in receptive field sizes at either spinal or supraspinal levels would serve to enhance spatial acuity but would reduce the total population output of nociceptive neurons; this may explain decreases in perceived pain intensity and pain intensity-related brain activation.

The absence of activation in SI and the deactivation of SII during the discrimination period does not mean that these regions are not important for spatial discrimination. SI nociceptive neurons have small receptive fields that can provide information about stimulus location (Kenshalo and Isensee, 1983). SI and SII are both somatotopically organized (Bingel et al., 2004; Mazzola et al., 2006). Lesions of SII lead to deficits in both tactile and pain discrimination and suggest that SII and SI may function together to process discriminative aspects of somatosensation (Murray and Mishkin, 1984; Greenspan and Winfield, 1992). However, electrophysiological and imaging studies indicate that although SI and SII may be necessary for spatial awareness, by themselves they are not sufficient to support this process (Laureys et al., 2002; de Lafuente and Romo, 2005). The involvement of other brain regions appears to be necessary for spatial awareness.

\section{Spatial discrimination-related activation}

Cerebral cortical regions including the right DLPFC, PPC, ACC, and associated regions of the right anterior and midline thalamus
Table 1. Spatial memory-related and spatial discrimination-related activations for pain

\begin{tabular}{|c|c|c|}
\hline Region & Memory & Discrimination \\
\hline Left IFC & $3.71(568 ;-36,44,-18)$ & $(-)$ \\
\hline Left operculum & $2.72(568 ;-50,32,-8)$ & $(-)$ \\
\hline \multicolumn{3}{|l|}{ Thalamus } \\
\hline Right & $(-)$ & $3.52(383 ; 6,-26,4)$ \\
\hline Left & $(-)$ & $(-)$ \\
\hline \multicolumn{3}{|l|}{ Caudate nucleus } \\
\hline Right & $(-)$ & $3.36(383 ; 14,4,10)$ \\
\hline Left & $(-)$ & $(-)$ \\
\hline \multicolumn{3}{|l|}{ Anterior insula } \\
\hline Right & $(-)$ & $(-)$ \\
\hline Left & $(-)$ & $(-)$ \\
\hline \multicolumn{3}{|l|}{ DLPFC } \\
\hline Right & $(-)$ & $3.35(557 ; 40,40,24)$ \\
\hline Left & $3.6(568 ;-20,68,-8)$ & $(-)$ \\
\hline \multicolumn{3}{|l|}{ DFC } \\
\hline Right & $(-)$ & $4.08(557 ; 26,46,28)$ \\
\hline Left & $3.24(568 ;-50,18,30)$ & $(-)$ \\
\hline \multicolumn{3}{|c|}{ 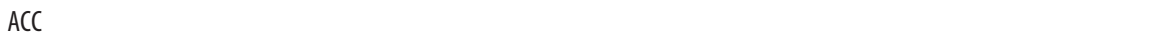 } \\
\hline Right & $(-)$ & $4.17(1557 ; 8,18,36)$ \\
\hline Left & $(-)$ & $4.06(1557 ;-2,38,26)$ \\
\hline Left M1/SI/PMA (arm) & $4.22(4244 ;-36,-38,40)$ & $5.35(3013 ;-32,-24,56)$ \\
\hline \multicolumn{3}{|l|}{ IPL } \\
\hline Right & $(-)$ & $4.33(3013 ; 44,-38,42)$ \\
\hline Left & $5.07(4244 ;-42,-54,50)$ & $4.88(944 ;-42,-34,42)$ \\
\hline \multicolumn{3}{|c|}{ 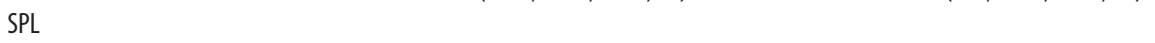 } \\
\hline Right & $(-)$ & $3.86(3013 ; 34,-50,52)$ \\
\hline Left & $(-)$ & $(-)$ \\
\hline \multicolumn{3}{|l|}{ SMA } \\
\hline Right & $(-)$ & $3.64(1557 ; 2,8,48)$ \\
\hline Left & $(-)$ & $4.23(1557 ;-6,-10,54)$ \\
\hline SI (leg) & $(-)$ & $(-)$ \\
\hline SII & $(-)$ & $(-)$ \\
\hline \multicolumn{3}{|l|}{ Deactivation } \\
\hline Left SII & & $3.56(545 ;-38,-24,22)$ \\
\hline Right anterior insula & & $4.26(717 ; 42,4,-14)$ \\
\hline Left posterior insula & & $3.77(545 ;-40,-16,0)$ \\
\hline
\end{tabular}

Peak Z scores were obtained from group analysis. Cluster sizes and peak locations are listed within parentheses as the number of voxels and $x, y, z$ coordinates (in millimeters) according to standard stereotaxic space. IFC, Inferior frontal cortex; DFC, dorsal frontal cortex; M1, primary motor cortex; PMA, premotor area; IPL, inferior parietal lobule; SPL, superior parietal lobule; SMA, supplementary motor area; -, no statistically reliable change.


Figure 5. Brain activation during the memory period is related to the use of verbal mnemonic strategies. In $>80 \%$ of discrimination trials, subjects used verbal strategies to remember the spatial location of the T1 stimulus. Consistent with such a verbal strategy, memory-related brain activation was lateralized to the left hemisphere in regions involved in verbal processing such as Broca's area on the inferior frontal gyrus. Activation during the memory period overlapped with activation during the discrimination period only in areas associated with motor planning. The left side of each brain image corresponds to the right side of the brain. IPL, Inferior parietal lobule; M1, primary motor cortex; DISCRIM., discrimination.

and right head of the caudate nucleus were differentially activated while subjects were comparing the spatial location of a noxious heat stimulus with that of a previously experienced stimulus (Fig. 3 ). These regions are active during processing of spatial aspects of multiple sensory modalities (Nobre et al., 1997; Bushara et al., 1999; Coghill et al., 2001). Moreover, lesions of the PPC, prefron- 
tal cortex, and occasionally of the cingulate cortex, thalamus, and basal ganglia can produce hemispatial unawareness and neglect of visual, auditory, and somatosensory stimuli. Similarly, transient disruption of the PPC has been shown to reduce awareness of somatic stimuli (Mesulam, 1981; Oliveri et al., 1999; Nager et al., 2004). In many instances, hemispatial neglect can occur in the absence of damage to primary sensory capabilities (Vallar, 1998), consistent with notions that full awareness of sensory features of pain requires interactions between SI and other brain areas (Coghill et al., 1994; Bushnell et al., 1999).

Four lines of evidence indicate that activation of these structures during spatial discrimination of pain is not related to affective processing. First, both $\mathrm{T} 1$ and $\mathrm{T} 2$ were of the same intensity $\left(49^{\circ} \mathrm{C}\right)$ and were delivered to similar body regions (lower leg) in close temporal $(30 \mathrm{~s})$ and spatial $(4-16 \mathrm{~cm})$ proximity. Thus, the affective content of the two stimuli was effectively identical, serving to minimize potential affective cues that could be used during the comparison of the two stimuli. Second, spatial discrimination of innocuous cool stimuli evoked pronounced activation of nearly identical regions of the ACC, also suggesting that discrimination-related activation of this structure is independent of the negative affect associated with painful heat. Third, discrimination-related activation of the ACC overlapped minimally with pain-related activation, consistent with evidence that this structure has functionally distinct subregions (Vogt, 2005). Finally, data acquired after subjects completed the discrimination task indicate that no areas activated during the process of discrimination remained differentially active. Thus, progressive sensitization over the course of sequential discrimination trials did not lead to progressively greater pain affect-related brain activation.

\section{Mechanisms supporting memory of spatial location}

In contrast to activation of this spatial network during the evaluation process, left lateralized regions involved in verbal processing, such as Broca's area, were activated while subjects were remembering the location of a noxious heat stimulus (Fig. 5). This activation is consistent with the high frequency of subjects' reports that they used a verbal strategy to remember the stimulus location and is consistent with previous psychophysical evidence that subjects use categorical strategies to remember features of a noxious stimulus (Rainville et al., 2004). Verbal processing areas in the left hemisphere were not active during the discrimination process. Thus, the categorical mnemonic representation of stimulus location may possibly be transformed into a nonverbal analog spatial memory at some point after the onset of the T2 stimulus. The right DLPFC has been repeatedly demonstrated to be involved in spatial working memory, is clearly activated during the predecision period, and thus may represent one site where this nonverbal memory is maintained for comparisons with incoming afferent information (Courtney et al., 1998). Studies examining expected pain indicate that this region, together with the ACC, exhibits activation that is related to expected sensory features of noxious stimuli and may hold mental representations of previously experienced stimuli (Koyama et al., 2005).

\section{The neural circuitry for spatial discrimination}

Convergence and subsequent comparisons between neural representations of recalled spatial locations and incoming afferent information about present stimulus location represent the fundamental computational process that leads to the formation of a perceptual decision in this delayed match-to-sample task. Anatomical evidence indicates that afferent sensory information can converge with information from spatial memory at multiple sites. Afferent somatosensory information is transferred in parallel from the SI and SII to the PPC (Neal et al., 1987). Conversely, mnemonic information can be transferred caudally from the DLPFC to the PPC (Selemon and Goldman-Rakic, 1988). Both the DLPFC and the PPC project to closely apposed regions of several other brain areas that are activated during the decisionmaking period. These regions include the ACC, anterior medial and medial dorsal nuclei of the thalamus, and head of the caudate nucleus (Selemon and Goldman-Rakic, 1985, 1988). Of these regions, the caudate nucleus represents one of the major input sites into basal ganglia-thalamocortical computational loops (Parent and Hazrati, 1995). These loops may serve to iteratively cycle recalled and incoming afferent information for sequential comparisons during the evaluation process.

Brain regions outside of the primary sensory cortices are critically involved in spatial awareness of visual and auditory information (Goodale and Milner, 1992; Ungerleider and Haxby, 1994; Romanski et al., 1999; Rauschecker and Tian, 2000; Anourova et al., 2001; Barrett and Hall, 2006). In addition to the present findings, converging lines of evidence indicate that structures beyond the SI contribute importantly to the conscious appreciation of a somatosensory stimulus. First, in patients in persistent vegetative states, the SI is robustly activated during noxious stimulation, whereas other regions involved in the processing of pain, such as the ACC and prefrontal cortex, remain inactive (Laureys et al., 2002). Second, electrophysiological studies indicate that monkeys do not perceive a tactile stimulus that is sufficient to activate neurons within the SI until neurons within the frontal cortex are also activated (de Lafuente and Romo, 2005). Finally, patients with prefrontal lobotomies have been reported to exhibit substantial alterations in their ability to appreciate the meaning and significance of their pain (Freeman and Watts, 1948).

In summary, the topographic information provided by SI and SII may be a necessary precursor for spatial discrimination of pain. However, much like the visual and auditory systems, discrimination of spatial features of pain involves significant frontoparietal interactions. The neural circuitry activated during the discrimination process can clearly support comparisons between previously experienced information and incoming afferent information. Moreover, by supporting such comparisons, this circuitry may provide a unique and highly personal historical context for the interpretation of sensory features of pain.

\section{References}

Anourova I, Nikouline VV, Ilmoniemi RJ, Hotta J, Aronen HJ, Carlson S (2001) Evidence for dissociation of spatial and nonspatial auditory information processing. NeuroImage 14:1268-1277.

Barrett DJ, Hall DA (2006) Response preferences for "what" and "where" in human non-primary auditory cortex. NeuroImage 32:968-977.

Bingel U, Lorenz J, Glauche V, Knab R, Glascher J, Weiller C, Buchel C (2004) Somatotopic organization of human somatosensory cortices for pain: a single trial fMRI study. NeuroImage 23:224-232.

Bushara KO, Weeks RA, Ishii K, Catalan MJ, Tian B, Rauschecker JP, Hallett M (1999) Modality-specific frontal and parietal areas for auditory and visual spatial localization in humans. Nat Neurosci 2:759-766.

Bushnell MC, Duncan GH, Hofbauer RK, Ha B, Chen JI, Carrier B (1999) Pain perception: is there a role for primary somatosensory cortex? Proc Natl Acad Sci USA 96:7705-7709.

Coghill RC, Sang CN, Maisog JM, Iadarola MJ (1999) Pain intensity processing within the human brain: a bilateral, distributed mechanism. J Neurophysiol 82:1934-1943.

Coghill RC, Gilron I, Iadarola MJ (2001) Hemispheric lateralization of somatosensory processing. J Neurophysiol 85:2602-2612.

Coghill RC, McHaffie JG, Yen YF (2003) Neural correlates of interindi- 
vidual differences in the subjective experience of pain. Proc Natl Acad Sci USA 100:8538-8542.

Courtney SM, Petit L, Maisog JM, Ungerleider LG, Haxby JV (1998) An area specialized for spatial working memory in human frontal cortex. Science 279:1347-1351

Crick F, Koch C (1995) Are we aware of neural activity in primary visual cortex? Nature 375:121-123.

de Lafuente V, Romo R (2005) Neuronal correlates of subjective sensory experience. Nat Neurosci 8:1698-1703.

Freeman W, Watts JW (1948) Pain mechanisms and the frontal lobes: a study of prefrontal lobotomy for intractable pain. Ann Intern Med 28:747-754.

Goodale MA, Milner AD (1992) Separate visual pathways for perception and action. Trends Neurosci 15:20-25.

Greenspan JD, Winfield JA (1992) Reversible pain and tactile deficits associated with a cerebral tumor compressing the posterior insula and parietal operculum. Pain 50:29-39.

Heekeren HR, Marrett S, Bandettini PA, Ungerleider LG (2004) A general mechanism for perceptual decision-making in the human brain. Nature 431:859-862.

Jenkinson M, Bannister P, Brady M, Smith S (2002) Improved optimization for the robust and accurate linear registration and motion correction of brain images. NeuroImage 17:825-841.

Kenshalo Jr DR, Isensee O (1983) Responses of primate SI cortical neurons to noxious stimuli. J Neurophysiol 50:1479-1496.

Koyama T, McHaffie JG, Laurienti PJ, Coghill RC (2005) The subjective experience of pain: where expectations become reality. Proc Natl Acad Sci USA 102:12950-12955.

Laureys S, Faymonville ME, Peigneux P, Damas P, Lambermont B, Del Fiore G, Degueldre C, Aerts J, Luxen A, Franck G, Lamy M, Moonen G, Maquet $P$ (2002) Cortical processing of noxious somatosensory stimuli in the persistent vegetative state. NeuroImage 17:732-741.

Mazzola L, Isnard J, Mauguiere F (2006) Somatosensory and pain responses to stimulation of the second somatosensory area (SII) in humans. A comparison with SI and insular responses. Cereb Cortex 16:960-968.

Melzack R, Casey KL (1968) Sensory, motivational, and central control determinants of pain. In: The skin senses (Kenshalo DR, ed), pp 423-435. Springfield, IL: Thomas.

Mesulam MM (1981) A cortical network for directed attention and unilateral neglect. Ann Neurol 10:309-325.

Murray EA, Mishkin M (1984) Relative contributions of SII and area 5 to tactile discrimination in monkeys. Behav Brain Res 11:67-83.

Nager W, Wolters C, Munte TF, Johannes S (2004) Transcranial magnetic stimulation to the parietal lobes reduces detection of contralateral somatosensory stimuli. Acta Neurol Scand 109:146-150.

Neal JW, Pearson RCA, Powell TPS (1987) The cortico-cortical connections of area 7b, PF, in the parietal lobe of the monkey. Brain Res 419:341-346.

Nobre AC, Sebestyen GN, Gitelman DR, Mesulam MM, Frackowiak RS, Frith CD (1997) Functional localization of the system for visuospatial attention using positron emission tomography. Brain 120:515-533.
Ogawa S, Lee TM, Kay AR, Tank DW (1990) Brain magnetic resonance imaging with contrast dependent on blood oxygenation. Proc Natl Acad Sci USA 87:9868-9872.

Oliveri M, Rossini PM, Pasqualetti P, Traversa R, Cicinelli P, Palmieri MG, Tomaiuolo F, Caltagirone C (1999) Interhemispheric asymmetries in the perception of unimanual and bimanual cutaneous stimuli. A study using transcranial magnetic stimulation. Brain 122:1721-1729.

Parent A, Hazrati LN (1995) Functional anatomy of the basal ganglia. I. The cortico-basal ganglia-thalamo-cortical loop. Brain Res Brain Res Rev 20:91-127.

Price DD, Bush FM, Long S, Harkins SW (1994) A comparison of pain measurement characteristics of mechanical visual analogue and simple numerical rating scales. Pain 56:217-226.

Rainville P, Doucet JC, Fortin MC, Duncan GH (2004) Rapid deterioration of pain sensory-discriminative information in short-term memory. Pain 110:605-615.

Rauschecker JP, Tian B (2000) Mechanisms and streams for processing of "what" and "where" in auditory cortex. Proc Natl Acad Sci USA 97:11800-11806.

Romanski LM, Tian B, Fritz J, Mishkin M, Goldman-Rakic PS, Rauschecker JP (1999) Dual streams of auditory afferents target multiple domains in the primate prefrontal cortex. Nat Neurosci 2:1131-1136.

Romo R, Salinas E (2001) Touch and go: decision-making mechanisms in somatosensation. Annu Rev Neurosci 24:107-137.

Romo R, Salinas E (2003) Flutter discrimination: neural codes, perception, memory and decision making. Nat Rev Neurosci 4:203-218.

Selemon LD, Goldman-Rakic PS (1985) Longitudinal topography and interdigitation of corticostriatal projections in the rhesus monkey. J Neurosci 5:776-794.

Selemon LD, Goldman-Rakic PS (1988) Common cortical and subcortical targets of the dorsolateral prefrontal and posterior parietal cortices in the rhesus monkey: evidence for a distributed neural network subserving spatially guided behavior. J Neurosci 8:4049-4068.

Talairach J, Tournoux P (1988) Co-planar stereotaxic atlas of the human brain. New York: Thieme.

Treede RD, Kenshalo DR, Gracely RH, Jones AK (1999) The cortical representation of pain. Pain 79:105-111.

Ungerleider LG, Haxby JV (1994) "What" and "where" in the human brain. Curr Opin Neurobiol 4:157-165.

Vallar G (1998) Spatial hemineglect in humans. Trends Cogn Sci 2:87-97.

Vogt BA (2005) Pain and emotion interactions in subregions of the cingulate gyrus. Nat Rev Neurosci 6:533-544.

Woolrich MW, Ripley BD, Brady M, Smith SM (2001) Temporal autocorrelation in univariate linear modeling of FMRI data. NeuroImage 14:1370-1386.

Worsley KJ, Evans AC, Marrett S, Neelin P (1992) A three-dimensional statistical analysis for CBF activation studies in human brain. J Cereb Blood Flow Metab 12:900-918. 